\title{
Clinical characteristics of 34 COVID-19 patients admitted to intensive care unit in Hangzhou, China*
}

\author{
Yi ZHENG ${ }^{1}$, Li-jun SUN ${ }^{2}$, Mi XU ${ }^{1}$, Jian PAN ${ }^{3}$, Yun-tao ZHANG ${ }^{1}$, \\ Xue-ling FANG ${ }^{1}$, Qiang $\mathrm{FANG}^{1}$, Hong-liu $\mathrm{CAI}^{\dagger \neq 1}$ \\ ${ }^{I}$ Department of Critical Care Medicine, the First Affiliated Hospital, School of Medicine, Zhejiang University, Hangzhou 310003, China \\ ${ }^{2}$ Respiratory Therapy Department, the First Affiliated Hospital, School of Medicine, Zhejiang University, Hangzhou 310003, China \\ ${ }^{3}$ Emergency Department, the First Affiliated Hospital, School of Medicine, Zhejiang University, Hangzhou 310003, China \\ †E-mail: 1193001@zju.edu.cn
}

Received Apr. 2, 2020; Revision accepted May 8, 2020; Crosschecked May 12, 2020

\begin{abstract}
Objective: This study summarizes and compares clinical and laboratory characteristics of 34 patients admitted to the intensive care unit (ICU) for complications from coronavirus disease 2019 (COVID-19) at the First Affiliated Hospital, School of Medicine, Zhejiang University, Hangzhou, China from Jan. 22 to Mar. 5, 2020. Methods: A total of 34 patients were divided into two groups, including those who required noninvasive ventilation (NIV) and invasive mechanical ventilation (IMV) with additional extracorporeal membrane oxygenation (ECMO) in 11 patients. Clinical features of COVID-19 patients were described and the parameters of clinical characteristics between the two groups were compared. Results: The rates of the acute cardiac and kidney complications were higher in IMV cases than those in NIV cases. Most patients had lymphocytopenia on admission, with lymphocyte levels dropping progressively on the following days, and the more severe lymphopenia developed in the IMV group. In both groups, T lymphocyte counts were below typical lower limit norms compared to B lymphocytes. On admission, both groups had higher than expected amounts of plasma interleukin-6 (IL-6), which over time declined more in NIV patients. The prothrombin time was increased and the levels of platelet, hemoglobin, blood urea nitrogen (BUN), D-dimer, lactate dehydrogenase (LDH), and IL-6 were higher in IMV cases compared with NIV cases during hospitalization. Conclusions: Data showed that the rates of complications, dynamics of lymphocytopenia, and changes in levels of platelet, hemoglobin, BUN, D-dimer, LDH and IL-6, and prothrombin time in these ICU patients were significantly different between IMV and NIV cases.
\end{abstract}

Key words: Coronavirus disease 2019 (COVID-19); Clinical characteristics; Intensive care unit (ICU); Mechanical ventilation

https://doi.org/10.1631/jzus.B2000174

CLC number: R511

\section{Introduction}

In December 2019, a series of patients became stricken with an acute respiratory illness of unknown cause, which has emerged in Wuhan, Hubei Province, China. Researchers now know these patients had

\footnotetext{
${ }^{\ddagger}$ Corresponding author

* Project supported by the Project for Emergency of Key R\&D Plan from Zhejiang Science and Technology Agency (No. 2020C03123), China (DD ORCID: Hong-liu CAI, https://orcid.org/0000-0003-3783-8328 (C) Zhejiang University and Springer-Verlag GmbH Germany, part of Springer Nature 2020
}

coronavirus disease 2019 (COVID-19) caused by severe acute respiratory syndrome coronavirus 2 (SARS-CoV-2) which has been classified in the $\beta$-coronavirus $2 \mathrm{~b}$ lineage. Within several months, this disease rapidly and widely spread in China and many other countries, causing an outbreak of COVID-19 worldwide.

By the end of April 2020, the cumulative number of confirmed cases of COVID-19 infections in China is more than 80000 and includes more than 4000 deaths, according to the notification from the National Health Commission of the People's Republic of China (NHC, 2020b). COVID-19 was the third lethal illness 
caused by a coronavirus, surpassed only by severe acute respiratory syndrome (SARS) (WHO, 2020) and Middle East respiratory syndrome (MERS) (WHO, 2019). About one third of COVID-19 inpatients were admitted to intensive care units (ICUs) in Wuhan, China (Huang et al., 2020) and doctors are recognizing that a critical ingredient in managing the impacts of the disease is effective treatment of severe and critical patients. Recent data (NHC, 2020b) showed that COVID-19 fatality rates in Hubei Province and outside of the province were significantly different, prompting researchers to seek more information about the characteristics and treatments of COVID-19 patients in ICUs. Deeper analyses of related data may help in developing more effective treatment protocols for future severe patients.

Here we describe clinical and laboratory characteristics, treatments, and outcomes of confirmed COVID-19 patients who were admitted to the ICU of a tertiary teaching hospital in Hangzhou (Zhejiang Province, China), about $775 \mathrm{~km}$ from the outbreak city of Wuhan. The Department of Critical Care Medicine in this hospital is a key clinical center at the national level and regional critical diagnosis and treatment center in Zhejiang Province. During the dates studied, nine severe COVID-19 patients were transferred from surrounding cities to the regional hospital and represent the surrounding population at a regional level.

\section{Subjects and methods}

\subsection{Participants and study design}

For this retrospective study, we analyzed data from patients admitted between Jan. 22 and Mar. 5, 2020, who had been diagnosed (according to the guidance of NHC (2020a)) with SARS-CoV-2 pneumonia in the ICU in the First Affiliated Hospital, School of Medicine, Zhejiang University, Hangzhou, China. This ICU and hospital had been designated for COVID-19 treatment and written informed consent was obtained from all participants in the study. To be included in this analysis, patients' data had to meet one of the specific conditions for admission to ICU including dyspnea and respiratory rate of $\geq 30$ times/min, oxygen saturation of $\leq 93 \%$ at rest without oxygen inhalation, $\mathrm{PaO}_{2} / \mathrm{FiO}_{2}$ $(\mathrm{P} / \mathrm{F})$ of $\leq 300 \mathrm{mmHg}(1 \mathrm{mmHg}=0.133 \mathrm{kPa})$, and other organ dysfunction such as shock.

\subsection{Data collection}

Two researchers independently reviewed patients' medical records during the period of study for epidemiological, demographic, clinical, laboratory, management, and outcome information. Disease onset was defined as the day that any related symptom was noticed by patients or family group members, as ascertained through researcher communications. Diagnoses were identified according to standard international definitions including: (1) acute respiratory distress syndrome (ARDS; Berlin definition) (Ranieri et al., 2012); (2) acute kidney injury (AKI; Kidney Disease: Improving Global Outcomes (KDIGO) Clinical Practice Guidelines) (Khwaja, 2012); (3) acute liver injury defined as an increase in alanine aminotransferase (ALT) over two times the upper limit of the normal range (ULN) or an increase in conjugated bilirubin or a combined increase in aspartate aminotransferase (AST), alkaline phosphatase and total bilirubin provided that one of them was above two times ULN (Ferrer et al., 2016); (4) cardiac injury following the definition described by Huang et al. (2020).

Noninvasive ventilation (NIV) included nasal oxygen therapy, mask oxygen inhalation, and highflow nasal cannula (HFNC). We recorded the duration of disease onset to first positive nucleic acid testing of respiratory tract specimen, ICU admission, ARDS, HFNC, invasive positive pressure ventilation (IPPV), and extracorporeal membrane oxygenation (ECMO).

\subsection{Laboratory confirmation and treatment}

Using the hospital laboratory, we tested patient blood including complete blood count, serum biochemistry, coagulation profiles, lactate dehydrogenase (LDH), and C-reactive protein. Plasma cytokines from all patients (interleukin-2 (IL-2), IL-4, IL-6, IL-10, tumor necrosis factor- $\alpha$ (TNF- $\alpha$ ), interferon- $\gamma$ $(\mathrm{IFN}-\gamma))$ were detected by enzyme-linked immunosorbent assay (ELISA) method according to the manufacturer's instructions (Elabscience Biotechnology Co., Ltd., Wuhan, China). T, natural killer (NK), and B lymphocyte cell counts were tested by flow cytometry. Respiratory specimens of all patients including sputum, pharyngeal swabs, bronchoalveolar lavage fluid, and bronchial aspirates were tested for SARS-CoV-2, using real-time reverse transcription polymerase chain reaction (RT-PCR) assays (Huang et al., 2020). 
Only one patient did not receive antiviral or systematic corticosteroid treatment. Thirty $(88.2 \%)$ were given empirical antibiotic treatment, and 27 (79.4\%) were given $\gamma$-globulin treatment. Indications for HFNC included $\mathrm{P} / \mathrm{F}$ of $<200 \mathrm{mmHg}$, resting respiratory rate of more than $>20$ per minute, and the patient presenting as conscious and cooperative. The timing of invasive ventilation included $\mathrm{P} / \mathrm{F}$ of $<150 \mathrm{mmHg}$, a resting respiratory rate of more than 30 per minute, and patients presenting as uncooperative, and/or onset of serious complications such as shock. Indications for ECMO (Brodie and Bacchetta, 2011) included severe hypoxemia (e.g., $\mathrm{P} / \mathrm{F}$ of $<80 \mathrm{mmHg}$, despite the application of high levels of positive end expiration pressure (typically $15-20 \mathrm{cmH}_{2} \mathrm{O}\left(1 \mathrm{cmH}_{2} \mathrm{O}=\right.$ $0.098 \mathrm{kPa})$ ) for at least $6 \mathrm{~h}$ in patients with potentially reversible respiratory failure, uncompensated hypercapnia with acidemia $(\mathrm{pH}<7.15)$ or excessively high end-inspiratory plateau pressure $\left(>35-45 \mathrm{cmH}_{2} \mathrm{O}\right.$, according to the patient's body size) despite the best accepted standard of care for management with a ventilator.

\subsection{Statistical analysis}

We compared clinical and laboratory data from the two groups. Continuous variables were described with medians in interquartile ranges (IQRs) and compared with the Mann-Whitney $U$ test. Two sets of data at different time points were compared using a twoway repeated measures analysis of variance (ANOVA) Categorical variables were described using frequency rates and percentages and compared by $\chi^{2}$ test or Fisher's exact test. A two-sided $\alpha$ of less than 0.05 was considered statistically significant. All analyses were performed using SPSS software (Version 22.0). The patients in our cohort were divided into cases only receiving NIV and cases requiring invasive mechanical ventilation (IMV), because the way of respiratory support was independently associated with the process and outcome (Lindenauer et al., 2014; Gacouin et al., 2015).

\section{Results}

None of the 34 patients included in this study was a resident of Wuhan City and none had direct exposure to the Huanan Seafood Market. Nine of the patients had been exposed to individuals with con- firmed SARS-CoV-2 infection or clustering onset and 17 had traveled within the epidemic area or had close contact with one or more people from the epidemic area. Eight patients had no history of any of the above. Other characteristics of study patients include (Fig. 1): (1) median duration from symptom onset to the first positive nucleic acid test was 4 (1-7) d; (2) median duration from symptom onset to ICU admission was 10.0 (7.0-11.3) d; (3) median duration from symptom onset to HFNC was $10(7-11) \mathrm{d}$ in 27 patients; (4) median duration from onset to IMV was $11(8-12) \mathrm{d}$ in 15 patients; (5) median duration from symptom onset to ECMO was 23 (18-29) d in 11 patients; (6) median age was $66(58-76)$ years and $23(67.6 \%)$ were men; (7) $24(70.6 \%)$ patients had chronic diseases including hypertension $(64.7 \%)$, diabetes $(23.5 \%)$, cardiovascular disease $(11.8 \%)$, chronic obstructive pulmonary disease $(5.9 \%)$, chronic liver disease $(11.8 \%)$, and chronic kidney disease (5.9\%).

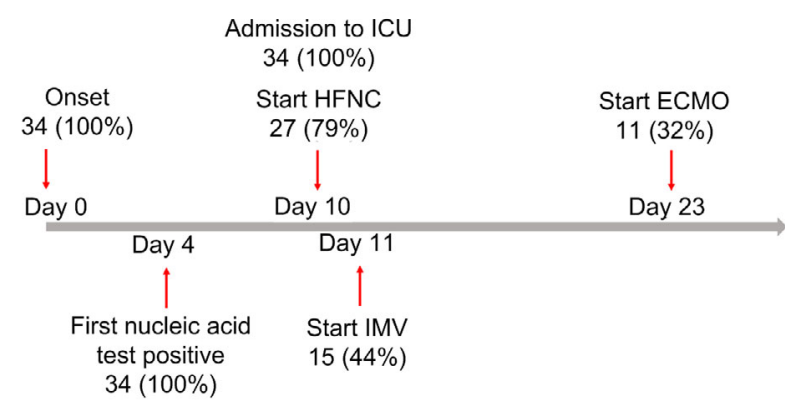

Fig. 1 Timeline of COVID-19 cases after onset of illness ICU: intensive care unit; HFNC: high-flow nasal cannula; ECMO: extracorporeal membrane oxygenation; IMV: invasive mechanical ventilation

The most common symptoms at onset of illness (Table 1) were fever (58.8\%), dry cough (20.6\%), and expectoration (29.4\%). Relatively less common initial symptoms were myalgia $(14.7 \%)$, fatigue $(5.9 \%)$, diarrhea $(5.9 \%)$, and headache $(5.9 \%)$. The proportion of different comorbidities and the distribution of first symptoms did not differ obviously.

The blood cell counts on admission showed that nearly half of the patients had leukocytosis (white blood cell count more than $10 \times 10^{9} \mathrm{~L}^{-1}, 15(44.1 \%)$ cases) while the incidence of leucopenia (white blood cell count less than $4 \times 10^{9} \mathrm{~L}^{-1}, 2(5.9 \%)$ cases) was very low. Lymphopenia (lymphocyte count less than $0.8 \times 10^{9} \mathrm{~L}^{-1}, 22(64.7 \%)$ patients) was present in more than half of the patients on admission. Platelet count 
Table 1 Demographics and baseline characteristics of patients with COVID-19

\begin{tabular}{|c|c|c|c|c|}
\hline Characteristics & $\begin{array}{c}\text { All cases } \\
(n=34)\end{array}$ & $\begin{array}{c}\text { IMV } \\
(n=15)\end{array}$ & $\begin{array}{c}\text { NIV } \\
(n=19)\end{array}$ & $\begin{array}{c}P \text { value } \\
\text { (IMV vs. NIV) }\end{array}$ \\
\hline Age (year) & $66(58,76)$ & $71(60,83)$ & $66(51,72)$ & 0.176 \\
\hline \multicolumn{5}{|l|}{ Sex } \\
\hline Male & $23(67.6 \%)$ & $11(73.3 \%)$ & $12(63.2 \%)$ & 0.715 \\
\hline Female & $11(32.4 \%)$ & $4(26.7 \%)$ & $7(36.8 \%)$ & 0.715 \\
\hline \multicolumn{5}{|l|}{ Comorbidities } \\
\hline Any & $24(70.6 \%)$ & $11(73.3 \%)$ & $13(68.4 \%)$ & 1.000 \\
\hline Hypertension & $22(64.7 \%)$ & $10(66.7 \%)$ & $12(63.2 \%)$ & 1.000 \\
\hline Diabetes & $8(23.5 \%)$ & $4(26.7 \%)$ & $4(21.1 \%)$ & 1.000 \\
\hline Cardiovascular disease & $4(11.8 \%)$ & $3(20.0 \%)$ & $1(5.3 \%)$ & 0.299 \\
\hline COPD & $2(5.9 \%)$ & $1(6.7 \%)$ & $1(5.3 \%)$ & 1.000 \\
\hline Chronic liver disease & $4(11.8 \%)$ & $1(6.7 \%)$ & $3(15.8 \%)$ & 0.613 \\
\hline Chronic kidney disease & $2(5.9 \%)$ & $1(6.7 \%)$ & $1(5.3 \%)$ & 1.000 \\
\hline \multicolumn{5}{|l|}{ Signs and symptoms } \\
\hline Fever & $20(58.8 \%)$ & $11(73.3 \%)$ & $9(47.4 \%)$ & 0.171 \\
\hline Dry cough & $7(20.6 \%)$ & $2(13.3 \%)$ & $5(26.3 \%)$ & 0.426 \\
\hline Expectoration & $10(29.4 \%)$ & $7(46.7 \%)$ & $3(15.8 \%)$ & 0.068 \\
\hline Fatigue & $2(5.9 \%)$ & $1(6.7 \%)$ & $1(5.3 \%)$ & 1.000 \\
\hline Diarrhea & $2(5.9 \%)$ & $0(0.0 \%)$ & $2(10.5 \%)$ & 0.492 \\
\hline Myalgia & $5(14.7 \%)$ & $2(13.3 \%)$ & $3(15.8 \%)$ & 1.000 \\
\hline Headache & $2(5.9 \%)$ & $2(13.3 \%)$ & $0(0.0 \%)$ & 0.187 \\
\hline \multicolumn{5}{|l|}{ Vital signs at ICU admission } \\
\hline Temperature $\left({ }^{\circ} \mathrm{C}\right)$ & $37.3(37.0,38.0)$ & $37.4(37.0,38.1)$ & $37.2(37.0,38.0)$ & 0.889 \\
\hline Respiratory rate $\left(\mathrm{min}^{-1}\right)$ & $23(19,29)$ & $25(22,32)$ & $20(16,26)$ & 0.060 \\
\hline Heart rate $\left(\min ^{-1}\right)$ & $79(67,88)$ & $67(62,91)$ & $80(70,87)$ & 0.509 \\
\hline MAP (mmHg) & $89(84,99)$ & $85(81,94)$ & $94(85,100)$ & 0.022 \\
\hline
\end{tabular}

Data are expressed as median (IQR) or number (percentage) of patients. $P$ values comparing NIV cases and IMV cases are from MannWhitney $U$ test or Fisher's exact test. IMV: invasive mechanical ventilation; NIV: noninvasive ventilation; COPD: chronic obstructive pulmonary disease; ICU: intensive care unit; MAP: mean arterial pressure; IQR: interquartile range

in the NIV group was higher than that in the IMV group, which were in normal range in most patients of both groups. As shown in Table 2, no significant differences were found between the NIV and IMV groups in terms of other biochemical indexes, inflammation or immune indicators, or coagulation indices on admission to hospital.

Eighteen patients $(52.9 \%)$ received HFNC without escalation of respiratory support. Fifteen $(44.1 \%)$ received IMV and 11 (32.4\%) patients required ECMO. Five $(14.7 \%)$ patients required continuous renal replacement therapy (CRRT). Common complications among the 34 ICU patients included ARDS (33 $(97.1 \%))$, acute liver injury $(14(41.2 \%))$, acute cardiac injury $(13(38.2 \%))$, and AKI (7 (20.6\%)). Table 3 shows that the complication rates (including acute cardiac injury and AKI) were significantly higher and discharge rate was notably lower in IMV than in NIV patients.

During the progression of the disease in this cohort of COVID-19 patients, we observed dynamic changes of the main laboratory indicators including changes in blood cell counts, biochemical parameters, coagulation profiles, and inflammatory cytokines every other day from Day 1 to Day 9 or discharged. White blood cell counts and neutrophil counts were at high levels during hospitalization, but for NIV patients were declining at the time of discharge. During hospitalization, most patients had marked lymphopenia, with IMV patients developing more severe 
Table 2 Laboratory findings in patients with COVID-19 on admission to hospital

\begin{tabular}{lccccc}
\hline \multicolumn{1}{c}{ Variables } & $\begin{array}{c}\text { Normal } \\
\text { range }\end{array}$ & $\begin{array}{c}\text { All patients } \\
(n=34)\end{array}$ & $\begin{array}{c}\text { IMV } \\
(n=15)\end{array}$ & $\begin{array}{c}\text { NIV } \\
(n=19)\end{array}$ & $\begin{array}{c}P \text { value } \\
\text { (IMV vs. NIV) }\end{array}$ \\
\hline WBC count $\left(\times 10^{9} \mathrm{~L}^{-1}\right)$ & $4.0-10.0$ & $8.9(5.3,14.3)$ & $6.2(4.4,13.5)$ & $9.3(6.6,16.0)$ & 0.160 \\
$\quad<4$ & & $2(5.9 \%)$ & $1(6.7 \%)$ & $1(5.3 \%)$ & 0.674 \\
$\quad 4-10$ & & $17(50.0 \%)$ & $8(53.3 \%)$ & $9(47.4 \%)$ & 0.674 \\
$\quad>10$ & $15(44.1 \%)$ & $6(40.0 \%)$ & $9(47.4 \%)$ & 0.674 \\
Neutrophil count $\left(\times 10^{9} \mathrm{~L}^{-1}\right)$ & $2.0-7.0$ & $7.8(4.3,13.2)$ & $5.7(3.6,12.8)$ & $8.0(5.5,14.8)$ & 0.223 \\
Lymphocyte count $\left(\times 10^{9} \mathrm{~L}^{-1}\right)$ & $0.8-4.0$ & $0.6(0.5,0.9)$ & $0.5(0.4,0.8)$ & $0.6(0.5,0.9)$ & 0.539 \\
$\quad<0.8$ & & $22(64.7 \%)$ & $10(66.7 \%)$ & $12(63.2 \%)$ & 1.000 \\
$\quad \geq 0.8$ & & $12(35.3 \%)$ & $5(33.3 \%)$ & $7(36.8 \%)$ & 1.000 \\
Platelet count $\left(\times 10^{9} \mathrm{~L}^{-1}\right)$ & $101-320$ & $178(147,196)$ & $156(111,176)$ & $191(172,201)$ & 0.012 \\
ALT $(\mathrm{U} / \mathrm{L})$ & $7-40$ & $20(15,30)$ & $21(14,31)$ & $19(15,27)$ & 0.768 \\
Total bilirubin $(\mu \mathrm{mol} / \mathrm{L})$ & $0.0-21.0$ & $12.0(7.6,18.6)$ & $11.8(7.3,18.5)$ & $12.1(8.0,18.7)$ & 0.876 \\
BUN $(\mathrm{mmol} / \mathrm{L})$ & $3.1-8.8$ & $7.0(5.4,10.5)$ & $7.5(6.0,14.2)$ & $6.6(5.0,8.1)$ & 0.111 \\
Creatinine $(\mu \mathrm{mol} / \mathrm{L})$ & $41-81$ & $84(67,104)$ & $84(67,106)$ & $84(67,99)$ & 0.445 \\
C-reactive protein $(\mathrm{mg} / \mathrm{L})$ & $0-8$ & $48(27,88)$ & $50(39,52)$ & $46(27,103)$ & 0.986 \\
D-dimer $(\mathrm{mg} / \mathrm{L})$ & $0-700$ & $606(383,1109)$ & $675(433,1613)$ & $467(370,1073)$ & 0.405 \\
IL-6 $(\mathrm{pg} / \mathrm{mL})$ & $0-7$ & $47(24,81)$ & $65(38,202)$ & $38(12,72)$ & 0.054 \\
IL-10 $(\mathrm{pg} / \mathrm{mL})$ & $0.0-2.3$ & $6.7(4.4,9.0)$ & $7.0(5.2,9.7)$ & $5.5(3.1,8.8)$ & 0.258 \\
\hline
\end{tabular}

Data are expressed as median (IQR) or number (percentage) of patients. $P$ values comparing NIV cases and IMV cases are from Mann-Whitney $U$ test or Fisher's exact test. IMV: invasive mechanical ventilation; NIV: noninvasive ventilation; WBC: white blood cell; ALT: alanine aminotransferase; BUN: blood urea nitrogen; IL: interleukin; IQR: interquartile range

Table 3 Treatments, complications, and outcomes in patients with COVID-19 during hospitalization

\begin{tabular}{lcccc}
\hline \multicolumn{1}{c}{ Parameter } & $\begin{array}{c}\text { All cases } \\
(n=34)\end{array}$ & $\begin{array}{c}\text { IMV } \\
(n=15)\end{array}$ & $\begin{array}{c}\text { NIV } \\
(n=19)\end{array}$ & $\begin{array}{c}P \text { value } \\
\text { (IMV vs. NIV) }\end{array}$ \\
\hline Treatments & $33(97.1 \%)$ & $15(100.0 \%)$ & $18(94.7 \%)$ & 0.5590 \\
Antiviral therapy & $33(97.1 \%)$ & $15(100.0 \%)$ & $18(94.7 \%)$ & 0.5590 \\
Glucocorticoid therapy & $30(88.2 \%)$ & $15(100.0 \%)$ & $15(78.9 \%)$ & 0.0840 \\
Antibiotics & $27(79.4 \%)$ & $14(93.3 \%)$ & $13(68.4 \%)$ & 0.0850 \\
$\gamma$-Globulin & $18(52.9 \%)$ & $0(0.0 \%)$ & $18(94.7 \%)$ & $<0.0001$ \\
HFNC & $15(44.1 \%)$ & $15(100.0 \%)$ & $0(0.0 \%)$ & $<0.0001$ \\
IMV & $11(32.4 \%)$ & $11(73.3 \%)$ & $0(0.0 \%)$ & $<0.0001$ \\
ECMO & $5(14.7 \%)$ & $4(26.7 \%)$ & $1(5.3 \%)$ & 0.1040 \\
CRRT & & & $18(94.7 \%)$ & 0.5590 \\
Complications & $33(97.1 \%)$ & $15(100.0 \%)$ & $1(5.3 \%)$ & 0.0190 \\
ARDS & $7(20.6 \%)$ & $6(40.0 \%)$ & $5(26.3 \%)$ & 0.0510 \\
AKI & $14(41.2 \%)$ & $9(60.0 \%)$ & $3(15.8 \%)$ & 0.0030 \\
Acute liver injury & $13(38.2 \%)$ & $10(66.7 \%)$ & & $<0.0001$ \\
Acute cardiac injury & & & $18(94.7 \%)$ & 1.0000 \\
Outcomes & $20(58.8 \%)$ & $2(13.3 \%)$ & $0(0.0 \%)$ & $0(0.0 \%)$ \\
Discharge & $0(0.0 \%)$ & $0(2 \%)$ & \\
Death & & & & \\
\hline
\end{tabular}

Data are expressed as number (percentage) of patients. $P$ values comparing NIV cases and IMV cases are from Fisher's exact test. IMV: invasive mechanical ventilation; NIV: noninvasive ventilation; HFNC: high-flow nasal cannula; ECMO: extracorporeal membrane oxygenation; CRRT: continuous renal replacement therapy; ARDS: acute respiratory distress syndrome; AKI: acute kidney injury 
lymphopenia over time. However, lymphocytes had returned to normal levels in NIV cases by the discharge day. Flow cytometry showed that median $\mathrm{T}$ lymphocyte counts were less than one third of the lower normal limit, compared to B lymphocytes in inpatients. T lymphocyte counts stayed low during hospitalization; however, the counts gradually rose to normal level until discharge in nearly half of NIV patients.

NK cell counts in more than $75 \%$ of patients were below the lower normal limit, and the counts declined progressively in IMV cases rather than in NIV cases during hospitalization. Platelet counts and hemoglobin levels were higher in NIV cases than in IMV cases, and hemoglobin levels dropped progressively in IMV cases during hospitalization. C-reactive protein levels in both groups showed gradual downward trend during hospitalization. ALT and D-dimer levels showed upward trends in both groups during hospitalization, while, for total bilirubin and blood urea nitrogen (BUN), the upward trends only occurred in IMV cases. The progressive decreases in LDH, IL-6, and IL-10 levels occurred in NIV cases rather than in IMV cases during hospitalization. The prothrombin time was increased, and BUN, D-dimer, LDH, and IL-6 levels were higher in IMV cases compared with NIV cases during hospitalization (Figs. 2 and 3).

\section{Discussion}

We reported 34 ICU patients with laboratoryconfirmed SARS-CoV-2 infection, characterized by severe or critical coronavirus pneumonia. Of the 34
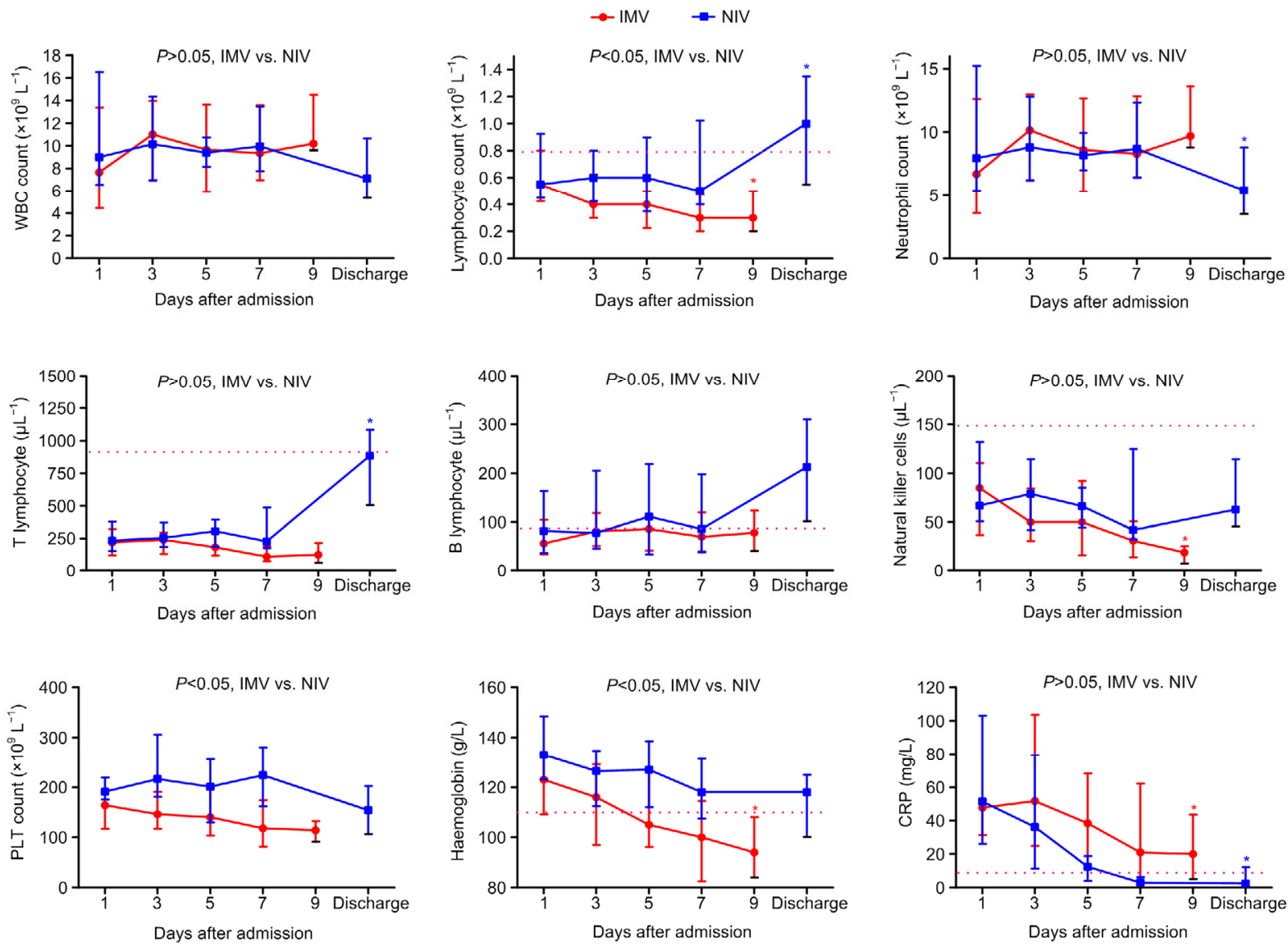

Fig. 2 Laboratory parameters (complete blood count and CRP) in 34 patients with COVID-19 (19 NIV cases and 15 IMV cases) every other day based on the days after admission

The dotted lines show the lower normal limits in lymphocyte, T lymphocyte, B lymphocyte, natural killer cell, and haemoglobin, and show the upper normal limit in CRP. Data are expressed as median (IQR). IMV: invasive mechanical ventilation; NIV: noninvasive ventilation; WBC: white blood cell; PLT: platelet; CRP: C-reactive protein; IQR: interquartile range. ${ }^{*} P<0.05$, vs. Day 1 within group 

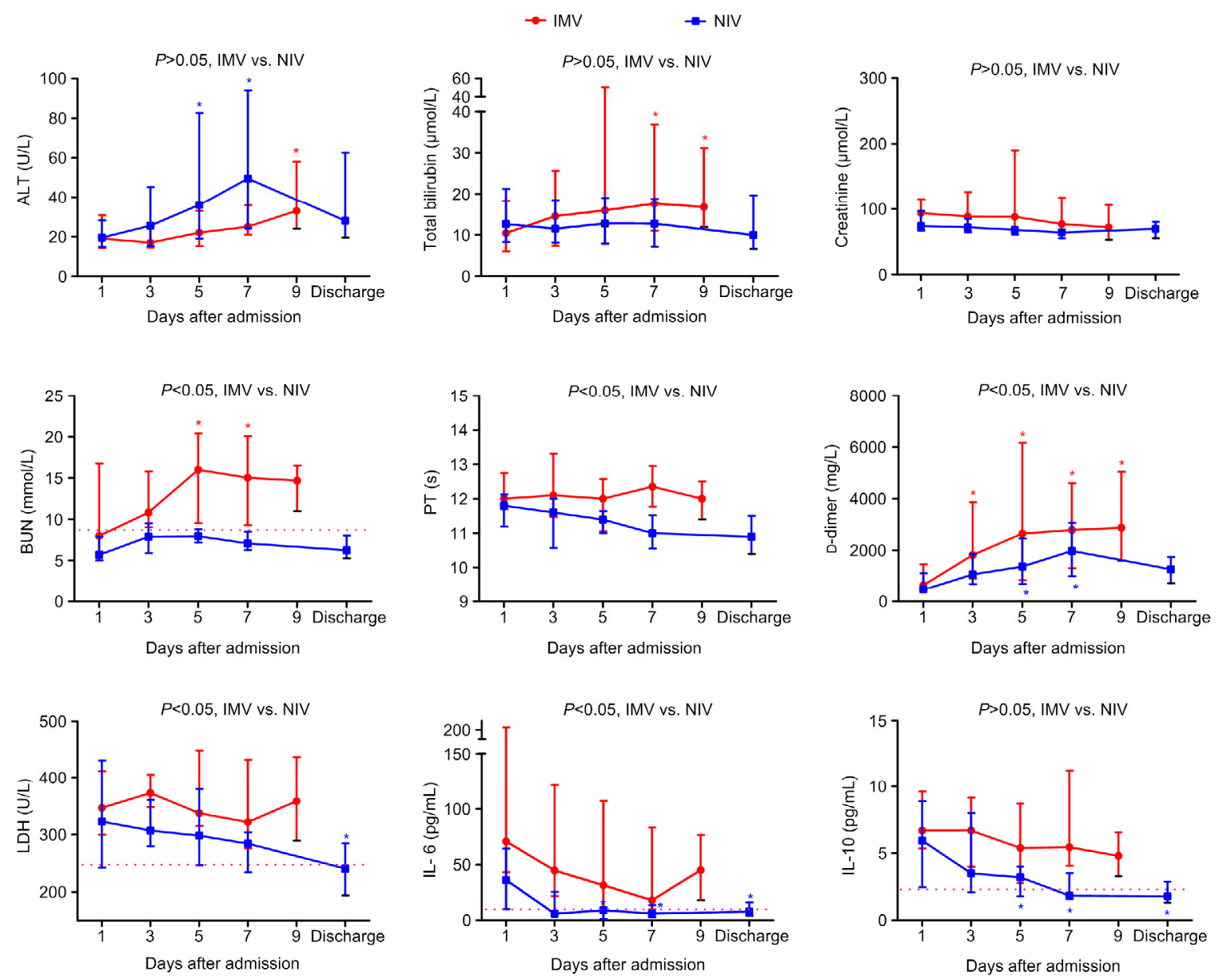

Fig. 3 Laboratory parameters (serum biochemistry and cytokines) in 34 patients with COVID-19 (19 NIV cases and 15 IMV cases) every other day based on the days after admission

The dotted lines show the upper normal limits in BUN, LDH, IL-6, and IL-10. Data are expressed as median (IQR). IMV: invasive mechanical ventilation; NIV: noninvasive ventilation; ALT: alanine aminotransferase; BUN: blood urea nitrogen; PT: prothrombin time; LDH: lactate dehydrogenase; IL: interleukin; IQR: interquartile range. ${ }^{*} P<0.05$, vs. Day 1 within group

patients, 19 patients $(55.9 \%)$ received noninvasive respiratory support (including nasal oxygen therapy in one case and HFNC in 18 patients) and $15(44.1 \%)$ required IMV. By Mar. 5, 2020, the rates of complications (e.g., acute liver, kidney, or cardiac injury) and discharge rates were significantly different between these two groups (a notable and positive outcome is that the oldest patient, a 94 years old man, was successfully treated and discharged).

More than 100 COVID-19 patients were admitted to the First Affiliated Hospital during our study period. Patients housed in the general isolation ward had mild to moderate symptoms, and as of Mar. 5, 2020, most ICU patients who did not require IMV have recovered and been discharged. However, by the end of Mar. 5, 2020, the majority of ICU patients requiring IMV are still receiving respiratory support including 11 patients who continue to require ECMO treatment. The high proportion of patients needing support in an ICU strongly suggests that a critical component to effectively fight COVID-19 and to protect the population will be to improve the management and treatment of severe and critical patients in hospital ICUs. This focus on effective hospital treatment for the critically ill is particularly important now because despite some progresses being made, to date no antiviral treatment has been proven to be effective for coronavirus infection.

In this study, the most common complication in patients was ARDS (33 (97.1\%) of 34 patients), underscoring the critical role of respiratory support in recovery. In this cohort, 33 infected patients received 
single or combined antiviral drugs including lopinavir/ ritonavir, baloxavir, favipiravir, darunavir, arbidol, and interferon- $\alpha$ inhalation. Corticosteroid and $\gamma$-globulin were administrated to about $80 \%$ of patients, but the efficacy of this treatment needs to be further confirmed.

Most patients in our cohort had neutrophilia, which is consistent with the results of SARS-CoVinfected patients as described by Wong et al. (2003). As mentioned in previous studies, for critically ill patients with SARS-CoV (Gu et al., 2005), SARSCoV-2 (Yang et al., 2020), and MERS (Chu et al., 2016; Liu et al., 2017) infection, lymphocytopenia is a prominent feature, indicating that targeted invasion by viral particles causes lymphocyte destruction. At admission to the hospital, $60 \%$ of the patients in our study already had lymphocytopenia and other patients in the study developed the condition as the disease progressed. In the IMV group, those with more severe illness, lymphocyte levels dropped progressively, and more severe lymphopenia occurred compared to the NIV group, suggesting that the severity of lymphocytopenia reflects the severity of SARS-CoV-2 infection. Research of SARS-CoV suggests that patients with more severe clinical illness and those who died had significantly more profound lymphopenia ( $\mathrm{He}$ et al., 2005). In our cohort, the lymphocyte counts in patients gradually rose as they recovered and were normal at time of discharge in the NIV group. These results differ from those of patients with SARS-CoV, in which lymphopenia was prolonged and lymphocyte levels did not return toward normal until patients were ill for five weeks (He et al., 2005). Our findings also differ from those of patients with H1N1 influenza A infection whose lymphocyte levels did not return to normal until 2-3 weeks after the disease onset (Cheng et al., 2019). Further analysis in our cohort showed that $\mathrm{T}$ lymphocytopenia was more pronounced than $\mathrm{B}$ lymphocytopenia, which was similar to previous study in SARS patients (Cui et al., 2003). The mechanism behind these dynamics is not clear and calls for further study and confirmation in cell and animal experiments.

We noted that SARS-CoV-2 infection caused increase in plasma IL-6 level, which was consistently at a high level in IMV cases in our cohort. Early studies also have shown increased concentrations of proinflammatory cytokines (e.g., IL- 6 , TNF- $\alpha$, and IFN- $\gamma$ ) in SARS (Wong et al., 2004) and MERS-CoV (Mahallawi et al., 2018) cases. Increased levels of anti-inflammatory cytokine, IL-10, in plasma were found in SARS-CoV-2-infected patients with IMV, which differed from SARS-CoV infection (Wong et al., 2003) but was supported by Huang et al. (2020) in patients with the same disease. BUN is a key element reflecting the intricate interrelation between nutritional status, protein metabolism, and renal situation of the patient (Arihan et al., 2018). BUN was significantly elevated in IMV patients compared with NIV cases, which may be caused by high catabolism. The characteristics of inflammation and metabolism suggested that we have to be on the alert of the existence of persistent inflammation-immunosuppression and catabolism syndrome (PICS) in COVID-19 patients with prolonged mechanical ventilation. PICS, often leading to secondary infections and viral reactivation in the critically ill, has been associated with increased morbidity and mortality (Limaye et al., 2008; Kalil and Florescu, 2009; Libert et al., 2015).

The baseline characteristics (including age, sex, and comorbidities) and laboratory indexes of patients in our cohort showed no statistical difference. However, the cases developed into different conditions needing different oxygen therapies and were complicated by injury to different organs impacting the probabilities and specifics of disease progression. Whether or not there are correlations or causal relationships among viral loads, lymphocytopenia, IMV, and organ injury remains to be verified by future research.

This study has several limitations. First, we had limited samples from our cohort to analyze and in places outside Hubei Province, and the total number of cases and the number of critical patients were relatively small, also limiting available samples to study. A previous report enrolled 52 critically ill patients with SARS-CoV-2 infection from Wuhan Jinyintan Hospital (Yang et al., 2020). Second, at the time of this writing, 13 IMV patients had not been discharged, so we cannot use survival or death as a clinical end point. The case mortality rate and other outcome indicators need to be reported later.

\section{Conclusions}

In this single-center case series of 34 ICU patients with SARS-CoV-2 infection in Hangzhou, China, 
$97.1 \%$ ( 33 cases) of patients had complications caused by ARDS, 44.1\% (15) received IMV, 55.9\% (19) only needed noninvasive respiratory support. Compared with cases in the NIV group, patients receiving IMV more readily developed complications from organ injury, had higher inflammatory markers, and developed more severe lymphopenia.

\section{Contributors}

Hong-liu CAI and Yi ZHENG conceived and designed the study, drafted the paper, and took responsibility for the integrity of the data and the accuracy of the data analysis. Li-jun SUN, Mi XU, and Jian PAN collected the data. Yi ZHENG, Xue-ling FANG, and Yun-tao ZHANG did the analysis. Qiang FANG revised the manuscript. All authors agree to be accountable for all aspects of the work and have read and approved the manuscript.

\section{Compliance with ethics guidelines}

Yi ZHENG, Li-jun SUN, Mi XU, Jian PAN, Yun-tao ZHANG, Xue-ling FANG, Qiang FANG, and Hong-liu CAI declare that they have no conflict of interest.

This study was approved by the National Health Commission of China and Ethics Commission of the First Affiliated Hospital, School of Medicine, Zhejiang University, Hangzhou, China (No. IIT20200077A). All procedures followed were in accordance with the ethical standards of the responsible committee on human experimentation (institutional and national) and with the Helsinki Declaration of 1975, as revised in 2008 (5). Informed consent was obtained from all patients for being included in the study.

\section{References}

Arihan O, Wernly B, Lichtenauer M, et al., 2018. Blood urea nitrogen (BUN) is independently associated with mortality in critically ill patients admitted to ICU. PLOS ONE, 13(1):e0191697. https://doi.org/10.1371/journal.pone.0191697

Brodie D, Bacchetta M, 2011. Extracorporeal membrane oxygenation for ARDS in adults. $N$ Engl $J$ Med, 365(20): 1905-1914. https://doi.org/10.1056/NEJMct1103720

Cheng YD, Zhao H, Song PX, et al., 2019. Dynamic changes of lymphocyte counts in adult patients with severe pandemic H1N1 influenza A. J Infect Public Health, 12(6): 878-883. https://doi.org/10.1016/j.jiph.2019.05.017

Chu H, Zhou J, Wong BHY, et al., 2016. Middle East respiratory syndrome coronavirus efficiently infects human primary $\mathrm{T}$ lymphocytes and activates the extrinsic and intrinsic apoptosis pathways. J Infect Dis, 213(6):904-914. https://doi.org/10.1093/infdis/jiv380

Cui W, Fan Y, Wu W, et al., 2003. Expression of lymphocytes and lymphocyte subsets in patients with severe acute respiratory syndrome. Clin Infect Dis, 37(6):857-859.

https://doi.org/10.1086/378587

Ferrer P, Amelio J, Ballarin E, et al., 2016. Systematic review and meta-analysis: macrolides- and amoxicillin/clavulanateinduced acute liver injury. Basic Clin Pharmacol Toxicol, 119(1):3-9. https://doi.org/10.1111/bcpt.12550

Gacouin A, Jouneau S, Letheulle J, et al., 2015. Trends in prevalence and prognosis in subjects with acute chronic respiratory failure treated with noninvasive and/or invasive ventilation. Respir Care, 60(2):210-218. https://doi.org/10.4187/respcare.03467

Gu J, Gong EC, Zhang B, et al., 2005. Multiple organ infection and the pathogenesis of SARS. J Exp Med, 202(3):415-424. https://doi.org/10.1084/jem.20050828

He ZP, Zhao CH, Dong QM, et al., 2005. Effects of severe acute respiratory syndrome (SARS) coronavirus infection on peripheral blood lymphocytes and their subsets. Int $J$ Infect Dis, 9(6):323-330. https://doi.org/10.1016/j.ijid.2004.07.014

Huang CL, Wang YM, Li XW, et al., 2020. Clinical features of patients infected with 2019 novel coronavirus in Wuhan, China. Lancet, 395(10223):497-506. https://doi.org/10.1016/S0140-6736(20)30183-5

Kalil AC, Florescu DF, 2009. Prevalence and mortality associated with cytomegalovirus infection in nonimmunosuppressed patients in the intensive care unit. Crit Care Med, 37(8):2350-2358. https://doi.org/10.1097/CCM.0b013e3181a3aa43

Khwaja A, 2012. Kdigo clinical practice guidelines for acute kidney injury. Nephron Clin Pract, 120(4):c179-c184. https://doi.org/10.1159/000339789

Libert N, Bigaillon C, Chargari C, et al., 2015. Epstein-Barr virus reactivation in critically ill immunocompetent patients. Biomed J, 38(1):70-76. https://doi.org/10.4103/2319-4170.132905

Limaye AP, Kirby KA, Rubenfeld GD, et al., 2008. Cytomegalovirus reactivation in critically ill immunocompetent patients. JAMA, 300(4):413-422. https://doi.org/10.1001/jama.300.4.413

Lindenauer PK, Stefan MS, Shieh MS, et al., 2014. Outcomes associated with invasive and noninvasive ventilation among patients hospitalized with exacerbations of chronic obstructive pulmonary disease. JAMA Intern Med, 174(12): 1982-1993. https://doi.org/10.1001/jamainternmed.2014.5430

Liu WJ, Zhao M, Liu KF, et al., 2017. T-cell immunity of SARS-CoV: implications for vaccine development against MERS-CoV. Antiviral Res, 137:82-92. https://doi.org/10.1016/j.antiviral.2016.11.006

Mahallawi WH, Khabour OF, Zhang QB, et al., 2018. MERS-CoV infection in humans is associated with a pro-inflammatory Th1 and Th17 cytokine profile. Cytokine, 104:8-13. https://doi.org/10.1016/j.cyto.2018.01.025

NHC (National Health Commission of the People's Republic of China), 2020a. Diagnosis and treatment of COVID-19 
(Version 6). http://www.nhc.gov.cn/xcs/zhengcwj/202002/ 8334a8326dd94d329df351d7da8aefc2/files/b218cfeb1bc 54639af227f922bf6b817.pdf [Accessed on Feb. 18, 2020] (in Chinese).

NHC, 2020b. Notification of 2020-NCOV infection. http://www. nhc.gov.cn/xcs/yqtb/202005/11f6b5e28be64f28b5b84ee d2984ed60.shtml [Accessed on Apr. 30, 2020] (in Chinese).

Ranieri VM, Rubenfeld GD, Thompson T, et al., 2012. Acute respiratory distress syndrome: the Berlin definition. JAMA, 307(23):2526-2533.

https://doi.org/10.1001/jama.2012.5669

WHO (World Health Organization), 2019. Middle East respiratory syndrome coronavirus (MERS-CoV). http://www. who.int/emergencies/mers-cov/en [Accessed on Mar. 5, 2020].

WHO, 2020. Summary of probable SARS cases with onset of illness from 1 November 2002 to 31 July 2003. https:// www.who.int/csr/sars/country/table2004_04_21/en [Accessed on Jan. 19, 2020].

Wong CK, Lam CWK, Wu AKL, et al., 2004. Plasma inflammatory cytokines and chemokines in severe acute respiratory syndrome. Clin Exp Immunol, 136(1):95-103. https://doi.org/10.1111/j.1365-2249.2004.02415.x

Wong RSM, Wu A, To KF, et al., 2003. Haematological manifestations in patients with severe acute respiratory syndrome: retrospective analysis. $B M J, 326(7403): 1358-1362$. https://doi.org/10.1136/bmj.326.7403.1358

Yang XB, Yu Y, Xu JQ, et al., 2020. Clinical course and outcomes of critically ill patients with SARS-CoV-2 pneumonia in Wuhan, China: a single-centered, retrospective, observational study. Lancet Respir Med, 8(5): 475-481.

https://doi.org/10.1016/S2213-2600(20)30079-5

\section{中文概要}

题 目: 中国杭州市重症监护室 34 例 COVID-19 患者临 床特点的分析

目 的: 本研究拟总结 2020 年 1 月 22 日至 3 月 5 日在中 国杭州浙江大学医学院附属第一医院重症监护 病房（ICU）收治的 34 例 2019 冠状病毒病 (COVID-19) 患者的临床和实验室特征。

创新点: 本研究比较了 COVID-19 患者有创机械通气组 (IMV) 与无创氧疗组 (NIV) 的临床和化验室 特征, 并探讨了两组患者间化验室指标的动态变 化的差异。

方 法: 纳入研究期间入住我院 ICU 的新冠肺炎重症患 者, 收集所有患者临床资料和化验室资料。根据 是否接受有创机械通气将患者分为 IMV 组和 NIV 组。比较两组的临床特征参数, 并动态观察 两组化验室指标的变化。

结 论: NIV 组患者急性肝、心、肾并发症发生率较高。 两组患者在入院时均出现淋巴细胞减少, 但随着 病情进展 IMV 组出现更严重的淋巴细胞减少症。 与 $\mathrm{B}$ 淋巴细胞相比, 两组 $\mathrm{T}$ 淋巴细胞计数均更明 显低于正常值下限。入院时, 两组患者的血浆白 介素-6（IL-6）水平均高于正常值上限。随着时 间的推移, NIV 组患者的 IL-6 水平下降更多。住 院期间 IMV 组患者的血小板、血红蛋白、血尿素 氮、凝血酶原时间、D-二聚体、乳酸脱氢酶和 IL-6 水平均高于 NIV 患者。

关键词：2019 冠状病毒病（COVID-19）；临床特征；重 症监护室 (ICU); 机械通气 